\title{
Investigation of Vertical Ground Reaction Force during Walking with the Exoskeleton for Patient with Unilateral Lower Limb Weakness
}

\author{
Niaam Kh. Al-Hayali ${ }^{1}$, Jumaa S. Chiad ${ }^{2}$, Somer M. Nacy ${ }^{3}$, and O. Hussein ${ }^{4}$ \\ \{neaam@kecbu.uobaghdad.edu.iq ${ }^{1}$,jumaachiad@gmail.com ${ }^{2}$, somernacy@googlemail.com ${ }^{3}$ \} \\ Biomedical Engineering Department, Alkhwarizmi College of Engineering, University of Baghdad ${ }^{1}$ \\ Mechanical Engineering Department, College of Engineering, Al-Nahrain University ${ }^{2}$ \\ Biomedical Engineering Department, Alkhwarizmi College of Engineering, University of Baghdad ${ }^{3}$
}

\begin{abstract}
Using lower extremity exoskeletons in the healthcare field like for rehabilitation is a substantial application. Lower limb exoskeletons can help in performing specific functions like gait assistance, and physical therapy support for patients who are lost their ability to walk again. The ground reaction force characteristics during human walking can be an important descriptor of gait disorders. The purpose of this study is to reveal the effect of the designed lower limb exoskeleton on the patient with right lower limb weakness based on the investigation of the ground reaction force. Results show that dynamic asymmetry practically disappears in the vertical GRF between the left and right legs during walking using the exoskeleton. The symmetry between the left and right limbs when walking using the exoskeleton for the maximum vertical GRF (first peak) is $98.9 \%$, the minimum vertical GRF (the valley) is $99 \%$, and the maximum vertical GRF (second peak) is $97.48 \%$.
\end{abstract}

Keywords: Exoskeleton, quasi-passive, ground reaction force, gait analysis, rehabilitation.

\section{Introduction}

Lower extremity exoskeletons have recently been extensively developed as helpful and rehabilitation aids for patients with lower-limb diseases [1-3]. Lower extremity exoskeletons may be defined as wearable systems that are worn by a patient and work in harmony with the patient's movements [4][5]. Lower extremity exoskeletons are used to assist rehabilitation training by providing functional motion assistance for patients with neurological and age-related disorders [3][6-8]. The features of ground reaction force (GRF) during human walking can be a useful diagnostic of abnormal gait. Exoskeletal ambulation can be investigated using GRF analysis to see how the exoskeleton affects the patient with lower limb weakness. The GRF acting on the body during upright movements is a major external force [9] which acts on the foot by a supporting horizontal surface during standing, walking, or running [10] with the same magnitude and in opposite direction to the force applied by the body [11] as shown in Fig 1. 


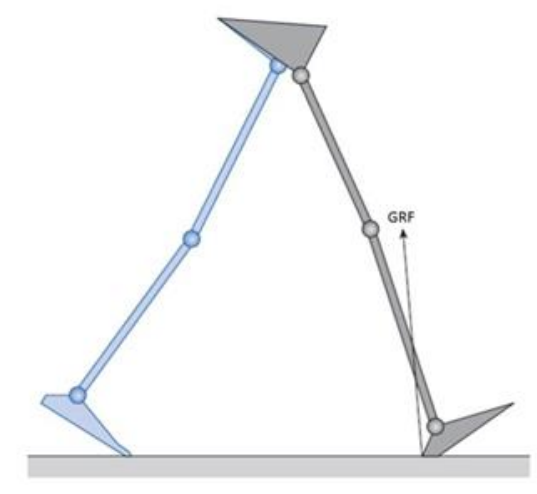

Fig. 1. GRF vector during the heel strike transient [12].

A mechanical sensor system [13] called a force plate is utilised to monitor GRF [14]. This plate is based on Newton's third law of motion [15][16]. Many professional and sophisticated computer systems include GRF measurement, which can be easily collected during normal clinical gait analysis [17]. This force is three-dimensional and consists of a single vertical [18] component plus two horizontal shear components [10]. Each component assesses a distinct aspect of the activity. The vertical component is mostly caused by the body's vertical acceleration [19]. The forces in the anterior-posterior and medial-lateral planes are represented by the two horizontal components [20]. The vertical component of the GRF, which is the force with the greatest magnitude that the earth reacts on the body, receives the most attention [21]. A typical vertical GRF profile of a single walking stride is shown in Fig 2. In a fraction of a second, the vertical GRF of contact with the ground heel strike (HS) will drop to zero and rise abruptly to virtually body weight. The body mass is travelling downhill and settling on the leg at the instant of foot flat (FF). It will be essential to apply a vertical force greater than body weight on the foot to decrease this downward motion while still supporting the body weight. The subject's foot is bearing 120 percent of his body weight at this time. The movement of the body's centre of mass is upward in mid-stance (MS). This action causes an upward acceleration, allowing the body to be supported by a force less than its own weight. At MS, this person has a body weight of 63 percent. The body mass is accelerated forward and upward at heel off (HO), preparing for the stance phase of the other leg. This indicates that the body will need to be supported by more than its own weight. Finally, toe-off (TO) is the point at which the foot loses contact with the ground and the force equalises. The M-shaped graph, also known as a double peak graph, depicts the fluctuation of force in relation to body weight and is typical of normal gait [22]. The purpose of this paper is to study the effect of the designed lower limb exoskeleton on a patient with lower limb weakness by investigated the vertical ground reaction force. 


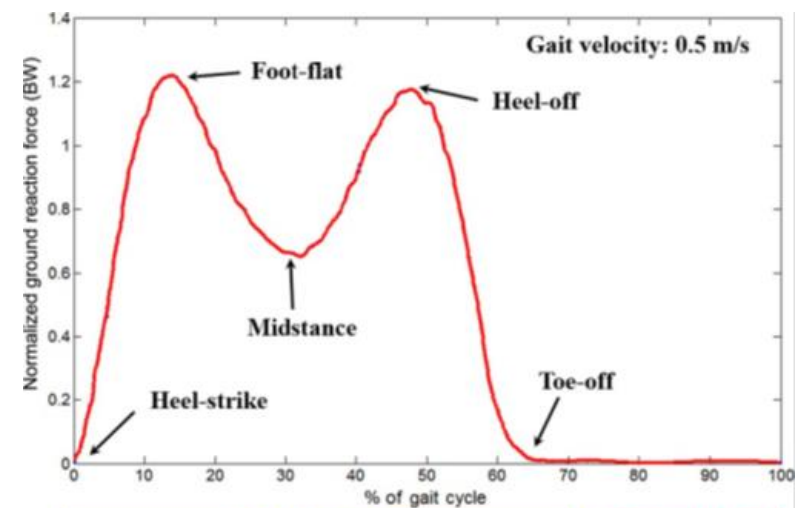

\begin{tabular}{l|l} 
R: Stance & $\mathbf{R}$ : Swing \\
\hline
\end{tabular}

Fig. 2. GRF in a normal walking stage [17].

\section{Materials and Methods}

\subsection{Subject}

Within Biomedical Engineering Department of the faculty of Al-Khawarizmi College of Engineering, University of Baghdad, all tests were carried out. The study was conducted on one subject, 21 years old female suffering from right side lower limb weakness. The weight of the subject was $(59 \mathrm{Kg})$, and the length was $(1.67 \mathrm{~m})$. The individual was given a thorough description of the study's purpose and process, as well as verbal instructions. The subject was chosen based on the projected exoskeleton's allowable length and shoe size range.

\subsection{Procedure}

Before the study began, the participant had approximately 15 minutes of acclimatization to the exoskeleton device. Later for walked subject, four or five tests were carried to ensure the exoskeleton with contented body fit. For providing rehabilitation of the right gait for the patient including to keep the control of the exoskeleton that might be difficult and thus sagittal plane motion is the most important plane during human walking is only considered and simplifies the hip joint and knee joint as one degree of freedom hinge. A 2 degree of freedom (DOF) quasipassive lower limb exoskeleton is designed to improve gait rehabilitation, as shown in Fig 3. In addition to its lightweight, the exoskeleton's links can be adjusted in size to align the exoskeleton's joints with the patient's joints. The exoskeleton's components were chosen after studying the kinetics and kinematics of human walking. 


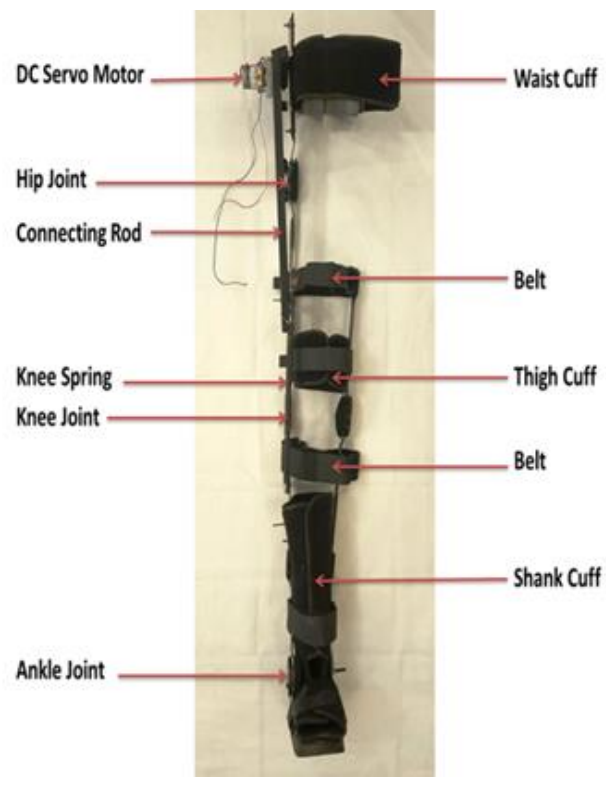

Fig. 3. The proposed lower limb exoskeleton components.

As demonstrated in Fig 4a, the patient begins walking without the exoskeleton at a sufficient distance from the force plate to achieve the self-selected speed before the foot contacts the force plate's centre. The ground reaction force was measured by the force plate as the walking procedure was repeated fifteen times. AMIT's Bioanalysis programme helped with the analysis, data processing, and evaluation. Soon these tests finished the trials, the truest data based on the intact left leg was chosen in the calculation results. The same experimental protocol was repeated for the patient when walking using the proposed exoskeleton as shown in Fig 4b.
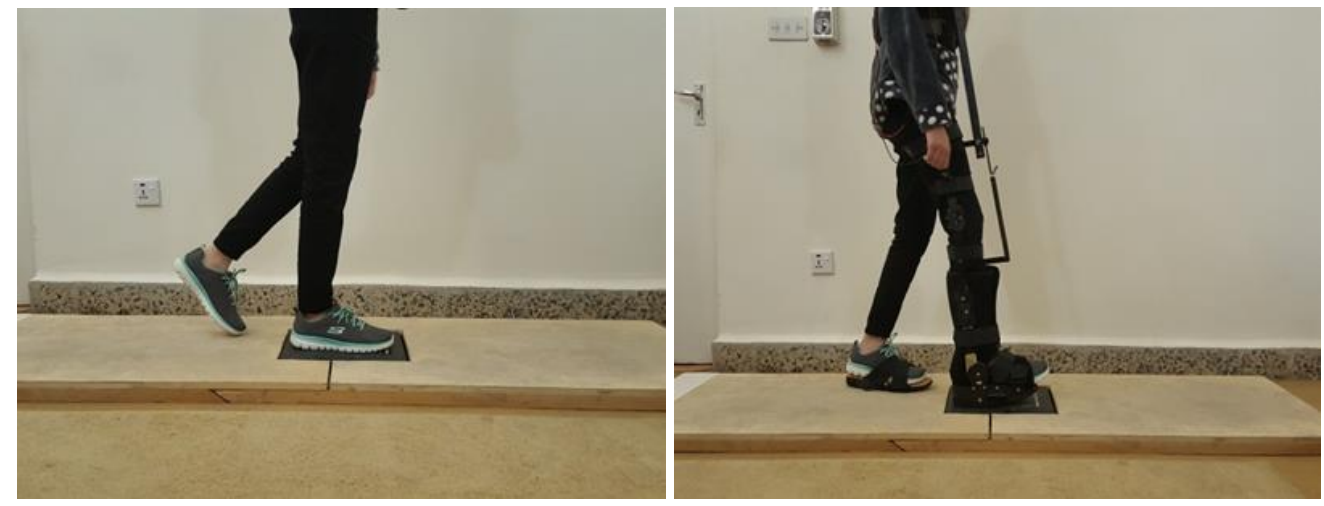

Fig. 4. The participant walking on a force plate (a) without the exoskeleton (b) using the exoskeletal device.

\section{Results and Discussion}


The ground reaction force has widely employed for medical and clinical tests for patient examination. The features of the GRF during human walking can be a useful diagnostic of abnormal gait. The variation of the vertical GRF for both limbs during walking without and with the designed exoskeleton is presented in Fig 5. The figure shows asymmetrical patterns in the vertical GRF of the $\mathrm{L}$ and $\mathrm{R}$ lower limb during the walking of the patient without using the exoskeleton. The time taken for posture stage is equal to $1.08 \mathrm{~s}$ and $1.1 \mathrm{~s}$ for left and right legs, respectively. The extreme values of the vertical GRF whe the legs are not considered the weights on L-R legs. The patient's left leg is normal and does not suffer from any problems; therefore, the vertical GRF for this leg has the normal two peaked form and the mid-stance valley. On moving downward and landed supported by the leg, the value of the first peak is equal to $457.69 \mathrm{~N}$, the mid-stance valley is $400 \mathrm{~N}$, and for forward and upward movements for the posture stage of the other leg, the second peak is equal to $445.25 \mathrm{~N}$. In comparison with the left leg, the weak right leg has a significant irregular reduction in all the extreme values of the vertical GRF. Shortly after the right heel strike and when the body mass moving downward, the vertical GRF rises to its first peak which is equal to $372.56 \mathrm{~N}$. The mid-stance has an irregular curve shape, and it has a hill with a peak value equal to $365.088 \mathrm{~N}$. The second elevation value during heel off is equal to $355.17 \mathrm{~N}$. When walking without using the exoskeleton, the symmetry between the two legs for the maximum vertical GRF (first peak) is $77.15 \%$, the minimum vertical GRF (the valley) is $90.44 \%$, and the maximum vertical GRF (second peak) is $74.64 \%$. Dynamic asymmetry practically disappears in the vertical GRF between the two lower limbs during walking using the exoskeleton. The duration of the stance phase for both legs is increased and is equal to $1.32 \mathrm{~s}$ for the left leg and $1.18 \mathrm{~s}$ for the right leg. Increasing stance duration when walking with the exoskeleton will provide static stability [23]. The extreme values of the vertical GRF of the right leg are increased and the curve shape has become close to normal. So, the value of the first peak comprises $423.9 \mathrm{~N}$ in L-Leg, $419.216 \mathrm{~N}$ in R-Leg, the value of the mid-stance valley is $396.7 \mathrm{~N}$ for L-Leg, $392.9 \mathrm{~N}$ for R-Leg, and the value of the second peak is $426.37 \mathrm{~N}$ for the left leg and $415.9 \mathrm{~N}$ for the right leg. For the vertical GRF, the symmetry between the left and right limbs when walking using the exoskeleton for the maximum vertical GRF (first peak) is $98.9 \%$, the minimum vertical GRF (the valley) is $99 \%$, and the maximum vertical GRF (second peak) is $97.48 \%$.

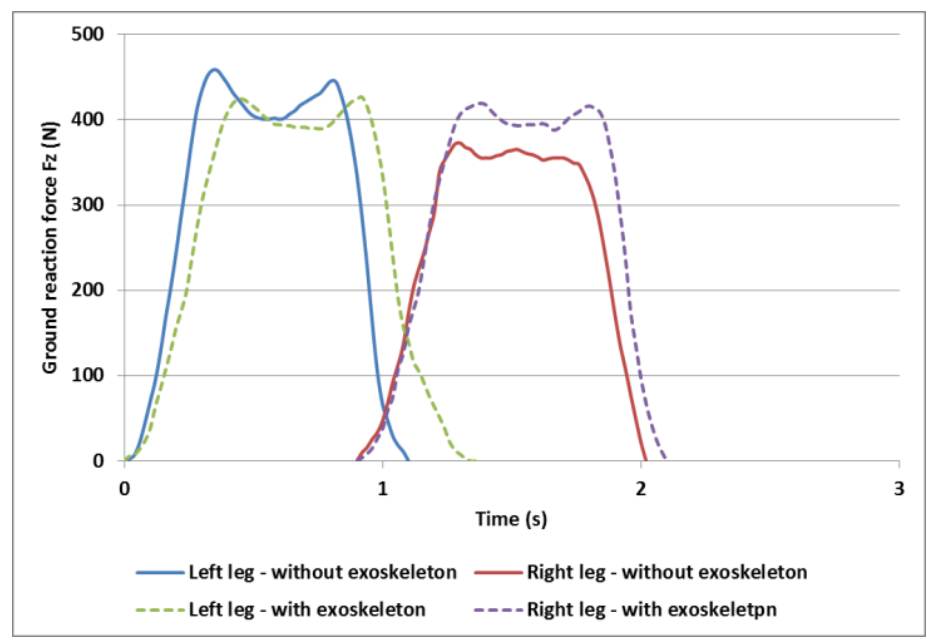


Fig. 5. Vertical walking GRFs of the subject with lower limb weakness before and after wearing the designed exoskeleton.

\section{Conclusion}

Ground reaction forces (GRFs) are employed to evaluate joint kinetics in biomechanical analysis, that mostly is considered to conclude many gait disorders. Investigating vertical ground reaction forces makes it possible to certain exoskeleton on people with lower limb weakness. The results show that the asymmetrical patterns in the vertical GRF between the left and the right leg disappear during walking using the designed exoskeleton which indicates that the designed exoskeleton help in performing gait improvement for a patient with unilateral lower limb weakness and providing static stability by increasing the stance duration.

\section{References}

1. M. Cestari, D. Sanz-Merodio, F.C. Arevalo, E G. ARES, a variable stiffness actuator with embedded force sensor for the ATLAS exoskeleton. Industrial Robot: An International Journal; 2014. p. 518-26.

2. Kong K, Jeon D. Design and control of an exoskeleton for the elderly and patients. IEEE/ASME Trans Mechatronics. 2006;11(4):428-32.

3. Sanchez-Villamañan MDC, Gonzalez-Vargas J, Torricelli D, Moreno JC, Pons JL. Compliant lower limb exoskeletons: A comprehensive review on mechanical design principles. J Neuroeng Rehabil. 2019;16(1):1-16.

4. Stopforth R. Customizable rehabilitation lower limb exoskeleton system. Int J Adv Robot Syst. 2012;9:1-7.

5. Krut S, Benoit M, Dombre E, Pierrot F. MoonWalker, a lower limb exoskeleton able to sustain bodyweight using a passive force balancer. Proc - IEEE Int Conf Robot Autom. 2010;2215-20.

6. Lyu M, Chen W, Ding X, Wang J, Bai S, Ren H. Design of a biologically inspired lower limb exoskeleton for human gait rehabilitation. Vol. 87, Review of Scientific Instruments. American Institute of Physics Inc.; 2016.

7. Huo W, Mohammed S, Amirat Y, Kong K. Active Impedance Control of a lower limb exoskeleton to assist sit-to-stand movement. Proc - IEEE Int Conf Robot Autom. 2016;2016-June:3530-6.

8. Chen B, Ma H, Qin LY, Gao F, Chan KM, Law SW, et al. Recent developments and challenges of lower extremity exoskeletons. J Orthop Transl [Internet]. 2016;5:26-37. Available from: http://dx.doi.org/10.1016/j.jot.2015.09.007

9. Chockalingam N, Healy A, Needham R. Interpreting Ground Reaction Forces in Gait. Handb Hum Motion. 2017;

10. Winter DA. Biomechanics and Motor Control of Human Movement: Fourth Edition. Biomechanics and Motor Control of Human Movement: Fourth Edition. 2009. 1-370 p.

11. Beckham G, Suchomel T, Mizuguchi S. Force Plate Use in Performance Monitoring and Sport Science Testing. New Stud Athl. 2014;29(3):25-37.

12. Whittle M. Gait analysis : an introduction. Butterworth-Heinemann; 2007. 255 p.

13. Lamkin-Kennard KA, Popovic MB. Sensors: Natural and Synthetic Sensors [Internet]. Biomechatronics. Elsevier Inc.; 2019. 81-107 p. Available from: 
http://dx.doi.org/10.1016/B978-0-12-812939-5.00004-5

14. Karatsidis A, Bellusci G, Schepers HM, de Zee M, Andersen MS, Veltink PH. Estimation of ground reaction forces and moments during gait using only inertial motion capture. Sensors (Switzerland). 2017;17(1):1-22.

15. Baldovino RG, Jamisola RS. A Survey in the Different Designs and Control Systems of Powered Exoskeleton for Lower Extremities. J Mech Eng Biomech. 2017;1(4):103-15.

16. Knudson D. Fundamentals of Biomechanics. Second Edi. Springer Science+Business Media, LLC. 2007.

17. Winiarski S, Rutkowska-Kucharska A. Estimated ground reaction force in normal and pathological gait. Acta Bioeng Biomech. 2009;11(1):53-60.

18. Fineberg DB, Asselin P, Harel NY, Agranova-Breyter I, Kornfeld SD, Bauman WA, et al. Vertical ground reaction force-based analysis of powered exoskeleton-assisted walking in persons with motor-complete paraplegia. J Spinal Cord Med. 2013;36(4):313-21.

19. Wardoyo S, Hutajulu PT, Togibasa O. A Development of Force Plate for Biomechanics Analysis of Standing and Walking. J Phys Conf Ser. 2016;739(1).

20. Headon R, Curwen R. Recognizing movements from the ground reaction force. ACM Int Conf Proceeding Ser. 2001;15-16-Nove:37.

21. Vaverka F, Elfmark M, Svoboda Z, Janura M. System of gait analysis based on ground reaction force assessment. Acta Gymnica [Internet]. 2015;45(4):187-93. Available from: https://doi.org/10.5507/ag.2015.022

22. Alamdari A, Krovi VN. A Review of Computational Musculoskeletal Analysis of Human Lower Extremities. Human Modeling for Bio-Inspired Robotics: Mechanical Engineering in Assistive Technologies. 2017. 37-73 p.

23. EV P. Investigation of biomechanical parameters of walking in the exoskeleton in patients with complete lower palsy. Int J Biosens Bioelectron. 2018;4(3):138-40. 